\title{
SPINAL BRUCELLOSIS
}

\author{
ROBERT M. LIFESO, EDWARD HARDER, SCOTT J. MCCORKELL
}

\author{
From the King Faisal Specialist Hospital and Research Centre, Riyadh
}

\begin{abstract}
Twenty-one patients with spinal brucellosis were reviewed. The disease is difficult to diagnose, and is often confused with spinal tuberculosis. Our study showed that it was best diagnosed by serology and bacterial culture; radiography and scanning were less helpful in the early stages.

After only six weeks' antibiotic treatment, there was a $55 \%$ clinical and serological reactivation rate: better results were achieved after at least three months of treatment. The adequacy of treatment was best monitored with repeated agglutination titres, and the duration of treatment proved to be more important than the antibiotic agent itself. Surgical intervention was reserved for biopsy, severe neurological impairment, or for spinal stabilisation.
\end{abstract}

Brucellosis remains a serious infectious disease and an important cause of morbidity in both animals and man. Despite intensive efforts at controlling the disease in animals (the source of human brucellosis), it is estimated that half a million people worldwide still contract brucellosis each year (Thimm 1982). In 1970325 cases of human brucellosis were reported from Scotland ( $\mathrm{Br} \mathrm{Med}$ $J$ 1971), and in the United States in 1979 the Communicable Disease Center reported 219 cases of human brucellosis (Mandell, Douglas and Bennett 1979). Countries reporting more than 1000 cases per year in their indigenous populations include Greece, Iran, Italy, Mexico, Peru and Spain ( JAMA 1983).

Brucellosis is predominantly due to three species of the genus Brucella: Brucella melitensis, first described by Marston in 1863 and the cause of disease in goats; Brucella abortus, the cause of bovine abortions described by Bang in 1897 and still endemic in 30\% of British dairy herds (Glasgow 1976); and Brucella suis, described by Tram in 1914. Since that time, Brucella canis and Brucella ovis have been described, although they rarely cause human disease.

Brucellae are small non-motile Gram-negative capnophilic coccobacilli that grow best at $37^{\circ} \mathrm{C}$ and at a pH range of 6.6-6.8. Brucellosis is found primarily in animals and is spread to humans by direct contact with infected tissue or by ingestion of infected animal products, most commonly milk or milk products ( $J A M A$ 1983). In western countries, brucellosis is an occupational disease found mainly in farmers, people working in meat packing plants, veterinary surgeons and livestock

R. M. Lifeso, FRCS (C), Chief, Division of Orthopaedic Surgery

E. Harder, FCRP (C). Chief, Infectious Diseases

S. J. McCorkell, MD, Department of Radiology

King Faisal Specialist Hospital and Research Centre, Riyadh 11211 , Kingdom of Saudi Arabia.

Requests for reprints should be sent to Dr R. M. Lifeso.

C 1985 British Editorial Society of Bone and Joint Surgery $0301-620 \mathrm{X} / 85 / 3083 \$ 2.00$ producers. In other areas of the world, the disease is more widespread and is also found in the general population.

The disease is characterised by fever, generalised malaise, night sweats, anorexia, weight loss, severe headache and polyarthralgia (Zammit-Maempel 1984). Physical findings depend on the duration of illness and can include hepatosplenomegaly, lymphadenopathy, spondylitis and arthropathy. Systemic brucellosis is occasionally complicated by meningitis, endocarditis, septic arthritis and osteomyelitis; such complications are, however, uncommon. The disease can evolve from the acute to a chronic state and may persist for many years.

The largest reported series of patients with brucellosis consisted of 6300 cases reported by Ganado and Craig (1958) from Malta: 699 of the patients reported had brucellosis involving bones or joints; 130 patients, or $2 \%$, had brucella spondylitis. Although spinal column involvement due to Brucella is rare, we decided nonetheless to review the literature as well as reporting a further 21 patients with brucella spondylitis.

\section{MATERIAL AND METHODS}

Patients. Between January 1976 and December 1983, 70 cases of brucellosis were treated at the King Faisal Specialist Hospital and Research Centre (Table I); 21 of these with spinal involvement form the basis of this report. The average duration of symptoms before presentation was 7.4 months (range 2 to 18 months). There were 20 men and one woman; their average age was 53.2 years (range 20 to 90 years).

The initial patient evaluation included a complete blood count, erythrocyte sedimentation rate (ESR), SMAC 20 (Technicon)* biochemical profile, electrocardiogram, chest radiograph and urinalysis. Serological studies included a brucelloslide test (Bio Merieux)

* SMAC 20: a sequential multiphasic analysis of chemistry done in a multichannel auto-analyser which simultaneously analyses 20 biochemical profiles. 
Table I. Brucellosis in 70 patients

\begin{tabular}{ll}
\hline Presenting complaint & Number of patients \\
\hline $\begin{array}{l}\text { Systemic (fever, arthralgia, weight loss, malaise, } \\
\text { headaches etc) }\end{array}$ & 25 \\
Spondylitis & 21 \\
Arthropathy & 12 \\
Central nervous system & 6 \\
Cerebrovascular system & 3 \\
Pleural effusion & 2 \\
Osteomyelitis & 1 \\
Total & 70 \\
\hline
\end{tabular}

followed by a brucella antibody tube dilution titre (Wellcome Diagnostics) to determine the antibody level in patients who were slide test positive. Plain radiographs of the spine were taken in all patients. Bone scans, computed tomography and myelography were performed in selected patients.

The diagnosis of brucella spondylitis was established by the following criteria: a minimum brucella antibody titre of $1: 80$; radiographic evidence of spinal involvement; and a clinical response to treatment. In addition to these criteria, seven patients had positive cultures for Brucella and in eight patients histological examination revealed a non-caseating granuloma without evidence of acid-fast bacilli.

Febrile patients had blood cultures drawn and incu- bated in a supplemental yeast peptone broth (BectonDickinson). The blood cultures, initially kept in a vacuum, were exposed to air on day 7 and were thereafter incubated in an atmosphere of $5 \%$ to $10 \% \mathrm{CO}_{2}$. Subcultures were made at weekly intervals onto chocolate blood agar plates and kept for four days. Synovial and surgical specimens were cultured in thioglycolate broth as well as being inoculated on routine culture media. All cultures identified as possibly containing fastidious organisms (those requiring specially enriched media and prolonged incubation) were routinely kept for 30 days. Cultures containing pale-staining, Gramnegative coccobacillary rods that were both oxidasepositive and urease-positive were identified as Brucella.

\section{RESULTS}

The average brucella titre in the patients with spondylitis was $1: 1280$, with a range of $1: 80$ to $1:>10000$. The lowest titre was $1: 80$, seen in one patient with positive blood cultures. The remaining patients all had titres equal to or greater than $1: 320$.

Blood cultures were positive in two patients, cultures obtained at operation were positive in three and cultures from joint fluid in two more; thus seven of the 21 patients had positive cultures. Other findings are shown in Table II.

Neurological involvement. A total of nine patients presented with varying degrees of neurological involvement which was always due to an extradural inflammatory mass: in no case was intradural extension of the disease found. One patient presented with a complete paraplegia at T3 and another with complete tetraplegia at C4; both

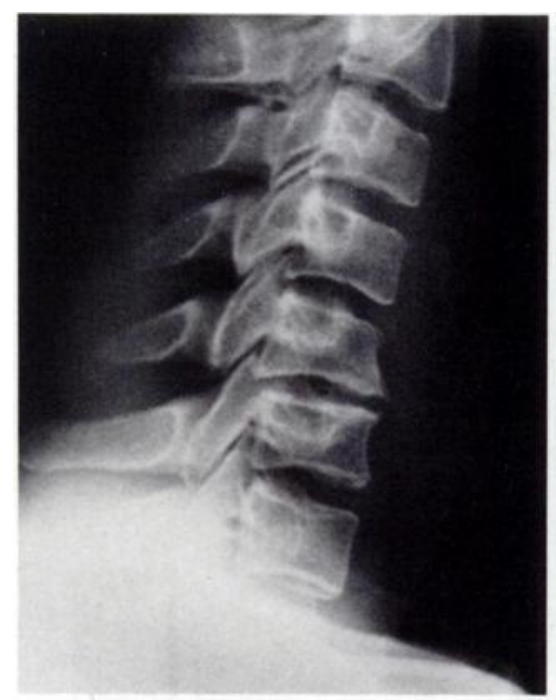

Fig. 1

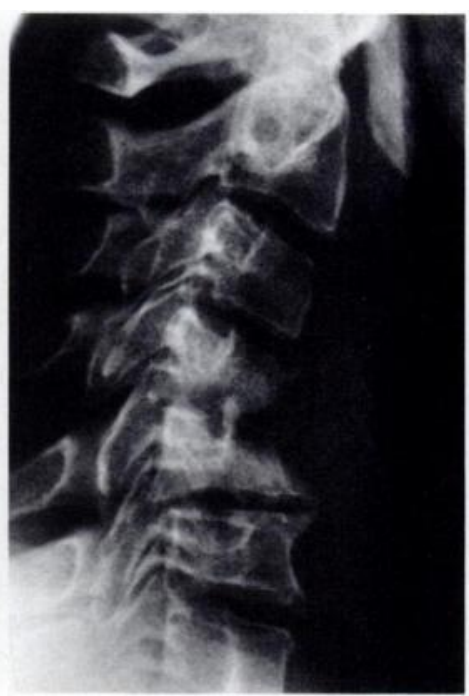

Fig. 2

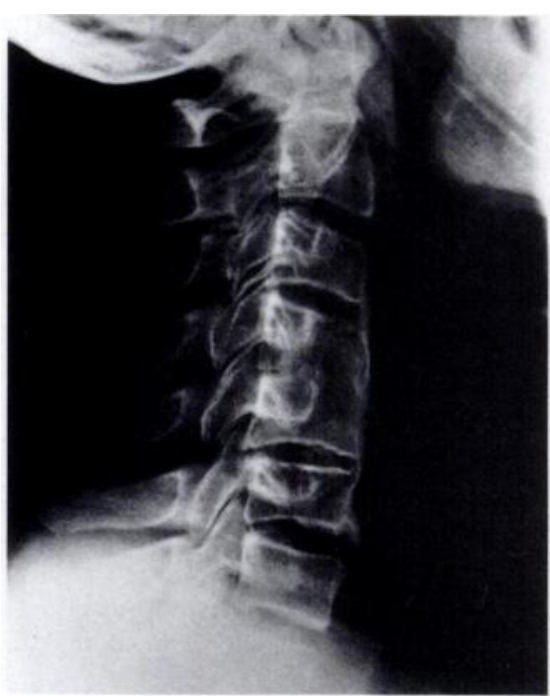

Fig. 3

A 40-year-old man presented with a four-month history of cervical spine pain and weakness in the left C5 root distribution. Figure 1 - Radiographs taken in November 1978 were interpreted as showing only $\mathrm{C} 5$ and $\mathrm{C} 6$ degenerative disc disease; in retrospect, a faint soft-tissue mass anterior to C4 and C5 was seen. Figure 2-By March 1979 a sclerotic destructive lesion and an increased soft-tissue prominence in front of the bodies of $\mathrm{C} 4$ and $\mathrm{C} 5$ were seen; physical examination revealed a $\mathrm{C} 5$ radiculopathy but no long tract signs. Figure $3-0$ cal discotomy and fusion at the C4-5 level a spontaneous fusion has occurred between C3-4, C6-7 and an early fusion is beginning at C7-T1. The retropharyngeal mass is absent and at this time there were no neurological abnormalities. 


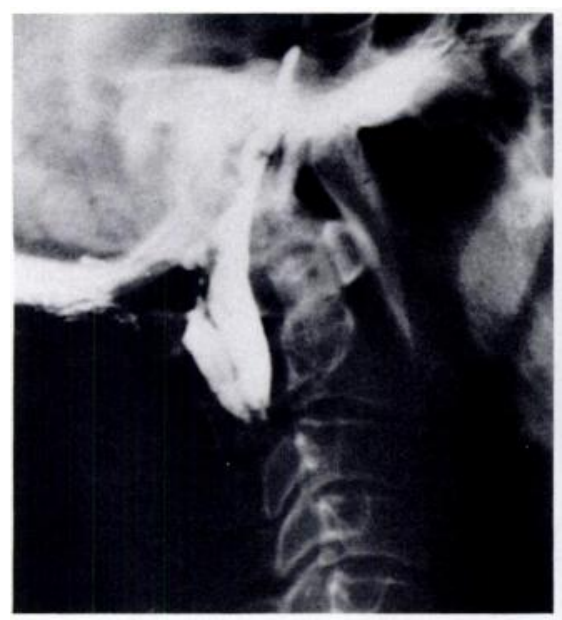

Fig. 4

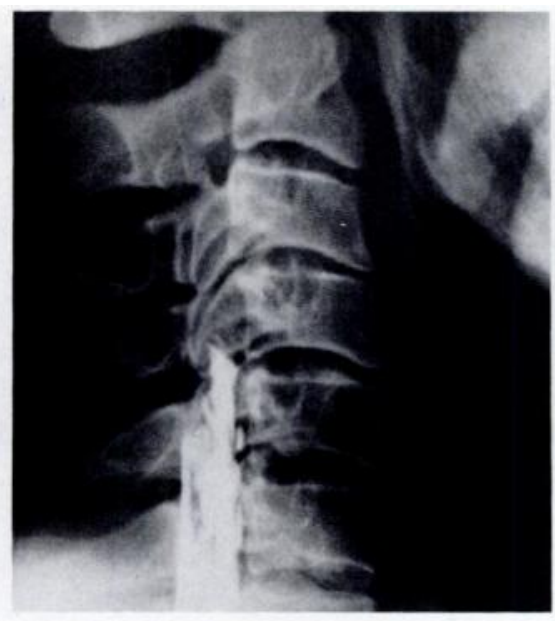

Fig. 5

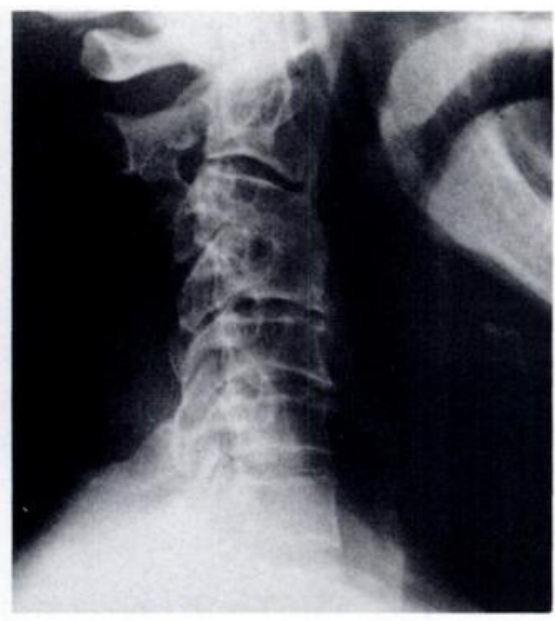

Fig. 6

A 55-year-old man presented with a five-day history of $\mathrm{C} 4$ tetraplegia. Figures 4 and 5 show cisternal and lumbar myelography. A complete extradural block extends from the inferior border of $\mathrm{C} 2$ to the superior border of $\mathrm{C} 5$. There is minimal disc space narrowing at C3-4 and a slight retropharyngeal soft tissue swelling. Figure 6-Nineteen months later. A posterior laminectomy for suspected intradural tumour revealed granulation tissue anterior to the dura. Subsequent anterior cervical decompression and fusion at C3-4 were performed. Spontaneous interbody fusion is occurring between $\mathrm{C} 2-3$ and $\mathrm{C}-5$. The patient is clinically well and neurologically intact.

underwent anterior spinal decompression after which they were able to walk. The remaining seven patients had incomplete neurological impairment on presentation, and four required surgical intervention: anterior decompression was performed in one, laminectomy in two, and fusion from the occiput to $\mathrm{C} 2$ in the remaining patient. Three achieved full recovery; one still walks with spasticity 20 months after laminectomy. Three patients were treated medically with resolution of their neurological symptoms. No patient worsened neurologically during the course of either surgical or medical treatment.

Radiological findings. On presentation nine patients $(42.8 \%)$ had no radiographic abnormalities, or subtle changes the significance of which was recognised only in retrospect. Two patients had slight retropharyngeal soft tissue swelling (Figs 1 and 4). Slight changes in the sacroiliac joints, difficult to distinguish from the early findings of a seronegative arthritis, were seen in two

Table II. Initial findings

\begin{tabular}{|c|c|c|}
\hline \multirow{2}{*}{$\frac{\text { Result }}{\text { Positive cultures }}$} & \multicolumn{2}{|c|}{ Number (\%) } \\
\hline & 7 & $(33)$ \\
\hline Neurological involvement & 9 & $(42.8)$ \\
\hline Recognisable spinal lesions & 12 & $(57.2)$ \\
\hline $\begin{array}{l}\text { Retropharyngeal swelling } \\
\text { Sacroiliac joint change } \\
\text { Joint space narrowing, osteophyte formation, sclerosis } \\
\text { No initial change }\end{array}$ & $\begin{array}{l}2 \\
2 \\
2 \\
3\end{array}$ & $(42.8)$ \\
\hline Hepatosplenomegaly & 6 & $(28.5)$ \\
\hline Adult onset diabetes & 3 & $(14.3)$ \\
\hline Renal failure & 1 & $(5.0)$ \\
\hline Chronic active hepatitis & 1 & $(5.0)$ \\
\hline
\end{tabular}

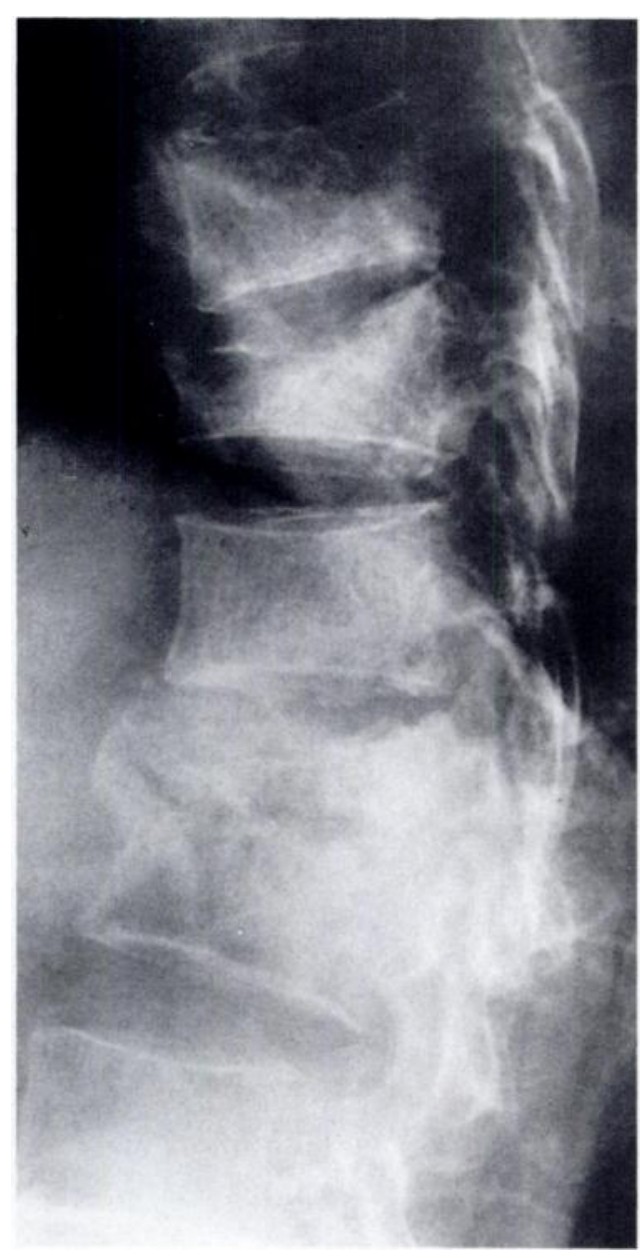

Fig. 7

A 53-year-old man with multiple levels involved and gross destruction of the vertebral bodies most noticeably at $\mathrm{LI}$ and T11. In spite of these changes, the patient was neurologically intact. 
patients, while two had disc space narrowing, osteophyte formation, and sclerosis which was initially attributed to osteoarthritis. The other three of these nine patients had no initial radiographic abnormalities.

Twelve patients $(57.2 \%)$ on presentation had radiographically recognisable lesions ranging from disc space narrowing with focal vertebral body endplate erosions to severe contiguous lesions involving up to four vertebral bodies and the intervening disc spaces. Altogether 29 discrete spinal lesions were found in the 21 patients. Four types of lesions were seen: a single lesion at one level (in

Table III. Distribution of disease (29 lesions in 21 patients)

\begin{tabular}{lcc} 
Location & Lesions \\
C1-C7 & \multicolumn{2}{c}{ Number $(\%)$} \\
T1-T12 & 6 & $(20.6)$ \\
L1-SI & 8 & $(27.6)$ \\
Sacroiliac joints & 13 & $(44.8)$ \\
\end{tabular}

12 patients); a single lesion involving multiple contiguous levels (4 patients); multiple lesions, each involving a single level (1 patient: see Fig. 7) and multiple lesions, each involving multiple contiguous levels (4 patients). Single intervertebral discs were involved in nine patients (Figs 1, 4 and 8), two patients had involvement of a single sacroiliac joint, and in one patient a single apophyseal joint was the site of the infection. Thirteen of the 29 lesions $(44.8 \%)$ occurred in the lumbar spine, eight $(27.6 \%)$ in the thoracic spine, six $(20.6 \%)$ in the cervical spine and two $(6.9 \%)$ in the sacroiliac joints (Table III).
Additional radiographic studies included bone scans which were positive in 12 patients and normal in one. Myelography was performed in the nine neurologically impaired patients. Total obstruction (of which an example is seen in Figs 4 and 5) was present in the two patients with clinical symptoms of paraplegia or tetraplegia and in one patient with midthoracic paraparesis. Extradural defects with incomplete obstruction were seen in the six other patients with less severe neurological symptoms. Myelographic findings were non-specific, but all revealed extradural compression.

CT scanning was performed in five patients; one such scan (Fig. 9) shows that this provides better visualisation of the spinal canal and adjacent soft tissues. Paravertebral soft tissue masses were seen in three patients, but were smaller and more localised than the soft tissue abscess usually associated with tuberculous spondylitis. In contrast to tuberculous spondylitis, no psoas abscesses were seen in any patient.

Healing was associated with bony ankylosis in $59 \%$ of lesions; an example is shown in Figure 3. In three patients ossification extended one disc space above the area of radiographically-involved bone, or one space below, or both. Twenty-nine per cent of lesions showed persistent disc space narrowing with remineralisation of previously demineralised or destroyed bone, as shown in Figure 12. Although prominent osteophytes formed in this group, there was no true bony ankylosis across the disc space. Twelve per cent of lesions showed no change over the period of follow-up.

Medical treatment. Twenty patients completed at least one course of supervised therapy while one patient took his medication in a sporadic fashion. Treatment was not standardised and was administered by different

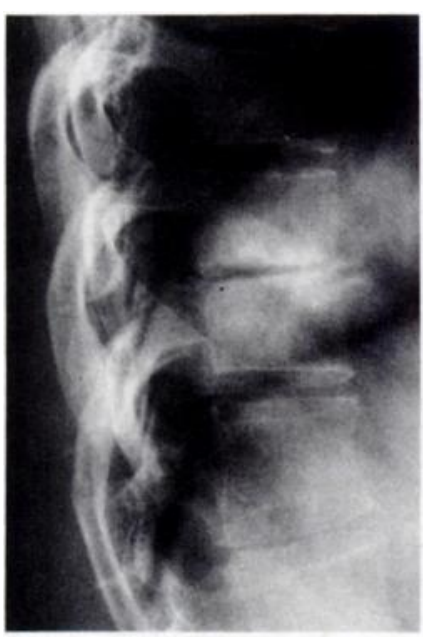

Fig. 8

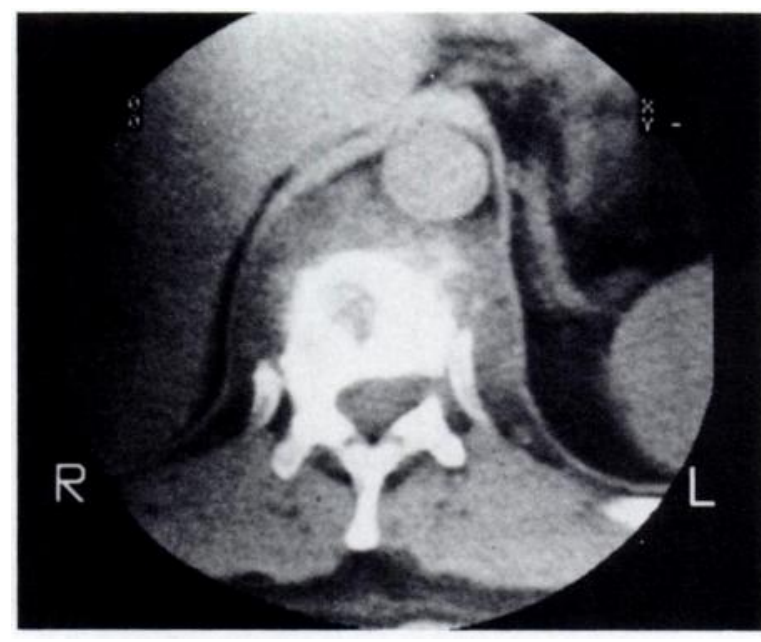

Fig. 9

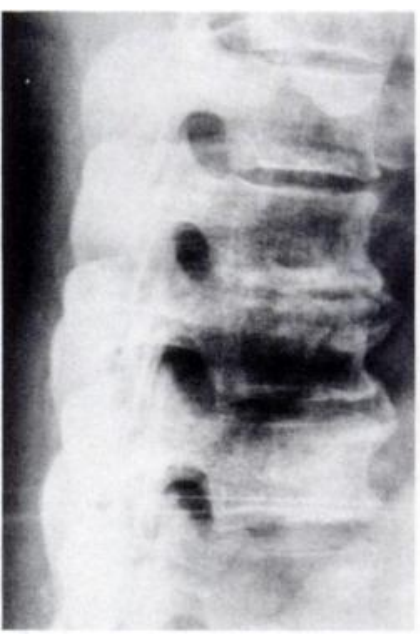

Fig. 10

A 55-year-old man with a two-month history of midthoracic pain and no evidence of neurological impairment. Figure 8 - The initial radiograph shows minimal disc narrowing. end-plate sclerosis and new bone formation behind the anterior longitudinal ligament. Figure 9 - The initial $C T$ scan reveals a large soft tissue mass anterior and lateral to the vertebral body with displacement of the aorta. Calcification can be seen in the soft tissue mass, originating from the anterior and lateral aspects of the body itself. Figure 10 One year later there is spontaneous fusion of the previously infected intervertebral space and the level below. The patient remained neurologically intact throughout his course of treatment. 


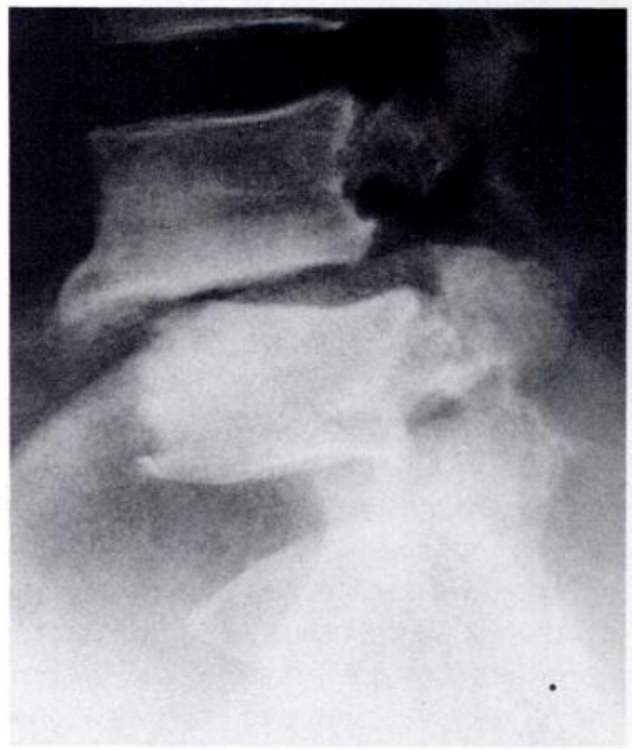

Fig. 11

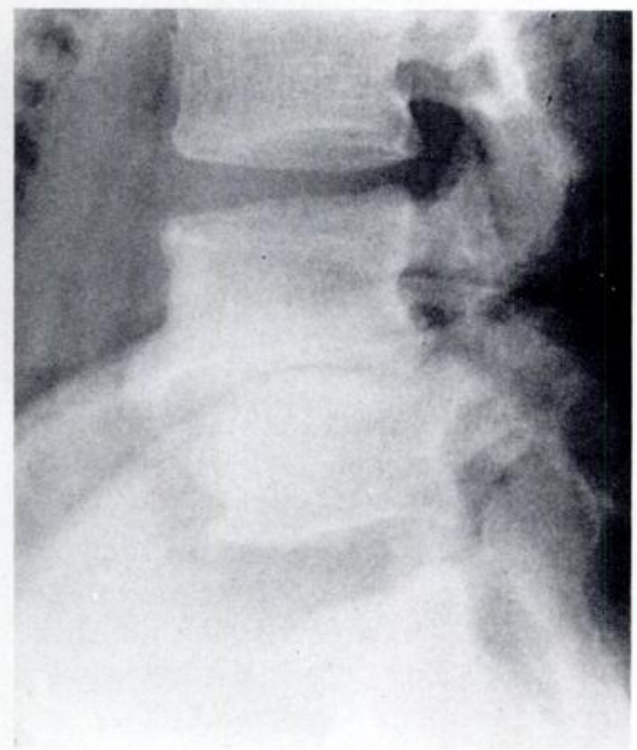

Fig. 12

A 55-year-old man was admitted in June 1982 unable to walk because of severe low back pain and bilateral weakness in the L5 root distribution. Bone scan showed increased activity at the L4-5 disc space. Aspiration of his right shoulder was positive for Brucella. Figure 11-Radiograph of lumbosacral spine on admission shows spondylolisthesis, anterior osteophytes at L4, disc narrowing at L4- 5 and anterior cortical erosions of L5. Figure 12-Radiograph 18 months later after a three-month course of co-trimoxazole. Neurological recovery has been complete, back pain is almost totally relieved and the radiograph shows complete disc obliteration, large anterior osteophyte formation and apparent stability at L4 and L5 with incomplete bony ankylosis.

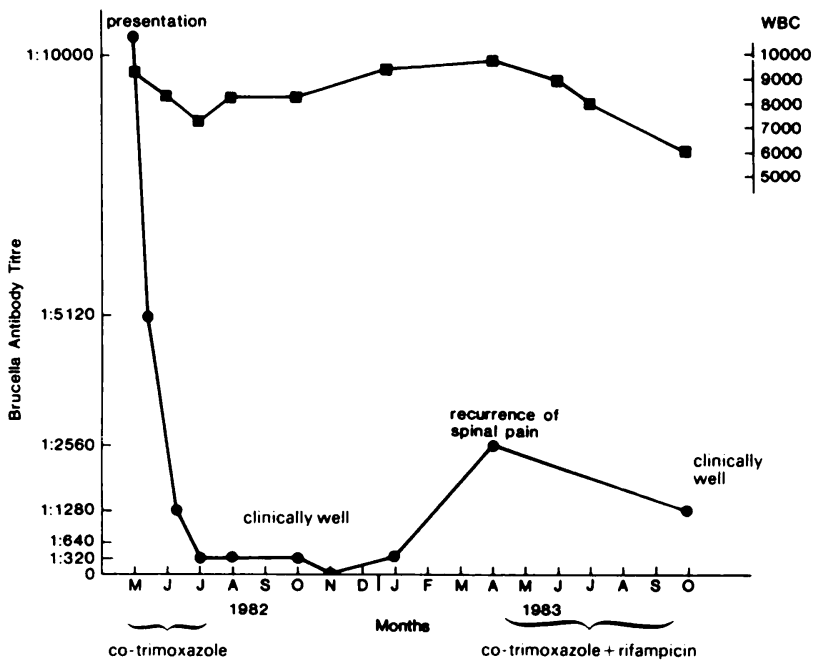

Fig. 13

A 62-year-old man presented with a two-month history of severe low back pain. Bone scan and radiograph showed a destructive lesion at L2-3. On presentation his brucella antibody titre was 1:10000. Treatment with co-trimoxazole, four tablets three times daily, gave rapid clinical improvement and was discontinued after 3 months. Nine months later he returned with a recurrence of severe lumbar pain and a brucella antibody titre of $1: 2560$. Treatment (co-trimoxazole and rifampicin) was resumed for six months until the titre had fallen to $1: 640$ and he had no symptoms thereafter. The white cell count during this period never exceeded 10000 . 
Table IV. Duration of primary course of drug treatment (20 patients*)

\begin{tabular}{lcl}
\hline Duration of treatment & Reactivated & Cured (follow-up) \\
\hline$<3$ months & 8 & $3(12$ months $)$ \\
$>3$ months & 3 & $6(23$ months $)$ \\
& 11 & 9 \\
\hline
\end{tabular}

* One patient excluded for poor compliance

physicians over the period of the review. It consisted of: a combination of tetracycline and streptomycin; co-trimoxazole (Septra, Septrin) alone; or a combination of co-trimoxazole and rifampicin.

Eleven of the 20 patients who completed a minimum 6-week course of therapy showed evidence of reactivation of their disease. There was no discernible difference in cure rate with any one of the drugs or combinations used. Eight of the 11 patients who relapsed following a primary course of drug treatment (Table IV) had less than 3 months of chemotherapy, whereas six of nine patients who were cured following their initial course of treatment had longer than 3 months. Relapse was judged by an increasing antibody titre and a recurrence of symptoms. The average follow-up was 19 months (range 12 to 50 months). Fourteen of the 20 patients were cured of their disease, nine after the first course of treatment and a further five after a second course of therapy. The remaining six patients continued with elevated brucella titres, although they were free of symptoms at review. Brucella antibody titres proved to be the most accurate way of assessing the effect of therapy (Fig. 13).

Surgical treatment. Ten of the 21 patients had surgical intervention. Six had surgical decompression and four had needle aspiration of the spine under radiographic control (Table V).

Anterior spinal decompression and fusion was performed in three patients. The indications were paraplegia in one, tetraplegia in another and severe midthoracic pain persisting despite 3 months of antibiotics in a third. All patients who had anterior decompression achieved solid fusion. Two patients underwent laminectomy for suspected intradural tumours; both were found to have granulation tissue surrounding the dura. Unlike tuberculosis, sequestration of bone or disc fragments was not found. One patient was fused from the occiput to $\mathrm{C} 2$ for gross destruction of the odontoid process with subluxation of $\mathrm{Cl}$ on $\mathrm{C} 2$.

\section{DISCUSSION}

Brucella spondylitis, first described in 1932 by Kulowski and Vinke (1932), is an extremely rare disease in man. The source of infection in our setting appeared to be the widespread use of unpasteurised camel or goat milk and milk products; the average age in our series was 53.2 years, whereas in the United Kingdom young adults are
Table V. Surgical intervention in 10 patients

\begin{tabular}{ll}
\hline Procedure & Number \\
\hline Anterior spinal decompression and fusion & 3 \\
Laminectomy & 2 \\
Occiput-C2 fusion & 1 \\
Needle aspiration & 4 \\
\hline
\end{tabular}

more often affected ( $\mathrm{Br}$ Med $J$ 1971). The incidence of spinal involvement was $30 \%$, which is higher than that reported by Ganado and Craig (1958). Diagnosis is difficult: the organism is fastidious and unless the disease is suspected and appropriate media are utilised, cultures are usually negative. The positive blood culture rate is less than 20\% (Wilson and Miles 1964). A positive culture, whether of joint fluid, blood or of infected bone or disc is diagnostic; however, the diagnosis is usually made by demonstrating a high or rising serum antibody titre to Brucella (Farrell, Robertson and Hinchliffe 1975).

Although bone involvement is the most frequent complication of chronic brucellosis (Jaffe 1972), ranging from $2 \%$ to $70 \%$ of reported cases, the earliest radiographic signs of spinal brucellosis are non-specific and may appear only months after the onset of symptoms. The earliest findings are minimal rarefaction of the contiguous vertebral end-plates, disc space narrowing, a small soft-tissue abscess, and occasionally anterior vertebral body erosions. A soft-tissue abscess, if present, is smaller than that usually seen in tuberculosis and does not involve the psoas. Often the early findings are recognised as being due to brucellosis only after bone scanning or the discovery of an elevated brucella antibody titre. With progression of the disease, there is increasing vertebral body destruction with sclerosis at the margins of the infective process. Eventually the spine stabilises with disc space narrowing, marked sclerosis, and osteophytes anterior and lateral to the involved disc space. In our patients bony ankylosis occurred in the majority of cases and only rarely were posterior elements of the spine involved. Large osteophytes and vertebral body sclerosis were usually prominent findings on late radiographs.

Treatment is usually conservative and consists of the appropriate antibiotic for at least 3 months. We have found that the duration of therapy is more important than the actual antibiotic agent itself. We now prefer co-trimoxazole or the combination of co-trimoxazole and rifampicin as our primary form of therapy (Daikos et al. 1973). If treatment is adequate, the systemic symptoms rapidly disappear and the brucella antibody titre will usually drop to $1: 160$ or less over a period of 3 to 4 months. We now maintain antibiotic cover until the titre is $1: 160$ or less and the clinical and radiographic evidence suggests a cure. A recurrence rate of $55 \%$ was seen following a primary course of treatment lasting only six 
weeks; as the symptoms and radiographic findings were always limited to the original site of disease, we suspected reactivation rather than reinfection. Recurrence of symptoms was controlled with reinstitution of co-trimoxazole and rifampicin.

\section{CONCLUSIONS}

Brucellosis occurs in 94 of the 153 countries of the world: the remaining countries have either already been successful in eradicating it or do not report its occurrence. In every case, man functions as a secondary host. Brucellosis, with its worldwide distribution, must be presumed to be present wherever cattle, sheep, goats, pigs or similar domestic animals are being kept by man, or where their products are being processed and utilised. The disease must be considered in the differential diagnosis of vertebral osteomyelitis. The initial radiographic findings are non-specific, and a high index of suspicion is necessary to make the diagnosis. Serum brucella agglutination titres are the most common method of diagnosis and the only reliable method of assessing the adequacy of treatment.

We have experienced a high rate of primary therapeutic failure with six weeks of therapy and now recommend a minimum course of three months, using a combination of co-trimoxazole and rifampicin. Treatment must be continued until the brucella agglutination titres have returned to $1: 160$ or less and clinical and radiographic evidence suggests a cure. Indications for surgery include: biopsy, decompression in the neurologically impaired patient and spinal stabilisation. The high recurrence rate is probably due to relapse, and careful long-term follow-up is strongly recommended.

The authors would like to thank Ms Nancy Napuunoa for her generous contribution in typing and revision of this manuscript.

\section{REFERENCES}

British Medical Journal. News and notes: epidemiology. Brucellosis. $\mathrm{Br}$ Med J 1971; iii: 197.

Daikos GK, Papapolyzos N, Marketos N, Mochlas S, Kastanakis S, Papasteriadis E. Trimethoprim-sulfamethoxazole in brucellosis. J Infect Dis 1973;128 Suppl:731-3.

Farrell ID, Robertson L, Hinchliffe PM. Serum antibody response in acute brucellosis. J Hyg (Camb) 1975;74:23-8.

Ganado W, Craig AJ. Brucellosis myelopathy. J Bone Joint Surg $[\mathrm{Am}]$ 1958;40-A:1380-8.

Glasgow MMS. Brucellosis of the spine. Br J Surg 1976;63:283-8.

Jaffe HL. Metabolic degenerative and inflammatory disease of bones and joints. Philadelphia: Lea \& Febiger, 1972.

Kulowski J, Vinke TH. Undulant (Malta) fever spondylitis: report of a case, due to Brucella melitensis, bovine variety, surgically treated. JAMA 1932;99: 1656-9.
Malhaven T. Zoonoses in modern medical practice. Henry Ford Hosp Med J 1982;30(1):4-6.

Mandell GL, Douglas RG, Bennett JE. Principles and practices of infectious disease. Chichester: John Wiley, 1979.

Journal of the American Medical Association. Leads from the Morbidity and Mortality Weekly Report; editorial. JAMA 1983;250:2600.

Thimm BM. Brucellosis: distribution in man, domestic and wild animals. Berlin: Springer, 1982.

Wilson GS, Miles AA. Topley and Wilson's principles of bacteriology and immunity. Sth ed. London: Edward Arnold, 1964.

Zammit-Maempel JV. Brucellosis. Med Int (Middle Eastern) 1984; Feb 2:85-8. 